\title{
A Novel Algorithm of Identification Theory of Complex Network for Public Transportation Network Cascading Failure
}

\author{
$\mathrm{Xu} \mathrm{Xu}$ \\ Business School of Shanghai Dianji University, Shanghai, 201306, China \\ Received: August 9, 2020. Revised: March 1, 2021. Accepted: March 23, 2021. Published: March 30, 2021.
}

\begin{abstract}
With the development of complex network theory and the gradual application of the traffic field, the problem of cascading failure has caused great attention of researchers. This paper tries to propose a new method based on complex network theory to measure the importance of nodes in the network. Based on complex network theory, this paper first discusses the network evolution mechanism of three main contents, define the importance of nodes in the network, and the design of the network center and the evaluation of the importance of node algorithm. In the end, a critical section identification method considering the failure probability and the failure consequence is designed, and the method for calculating the node importance based on the cascading failure is proposed. Using complex network theory, a quantitative assessment of the center of public transportation network and node importance model is designed. The bus network center, for the study of node importance analysis of bus network survivability has important significance. Help guide the optimization of public transport network service. Improve transport capacity of public transportation system.
\end{abstract}

Keywords-Complex network; Transportation network; Reliability; Importance degree; cascading failure

\section{INTRODUCTION}

I

$\mathrm{n}$ recent years, the traffic demand is increasing rapidly and

the road pressure doubled, but the traffic congestion is still

growing, and its outstanding performance is more and more traffic. More and more traffic participants are getting more and more [1-3].

The ultimate goal of urban transportation system planning is to meet the long-term development needs of urban economic and social, and to ensure the performance of the network system to achieve the best, to ensure that the traveler can easily, quickly, safely and comfortably. However road construction of our country long-term backward growth in traffic demand, road network structure are congenitally deficient, lack of necessary network reserve capacity, the network cannot adapt to unexpected situations, the robustness of the traffic planning scheme, still need to be improved. In practice, in order to improve the operation efficiency and reliability of the road network, it usually takes a lot of money to transform the existing network. If we can consider the uncertainty of traffic supply and demand in the road network planning and design phase, we can maximize the reasonable allocation of resources, avoid the waste of investment, so that the limited investment in the country can get the best investment benefit, but also can achieve the efficient matching of traffic supply and demand, avoid the occurrence of large area traffic jams [4-6].

Therefore, the reform of the traditional transportation planning method is based on public transportation network node importance measurement technology research, whether in theory or in engineering practice has a very important significance.

\section{COMPLEX NETWORK THEORY}

\section{A. The concept of complex networks}

It is very easy to generate rules and random networks. As a result, it is considered to be a reference to the two definitions of complex systems. The core of complex network is to explore the relationship between network structure and network function. Network structure is reflected in the network topology, the formation mechanism, the evolution law and network modeling. The network topological properties include the length of the characteristic path length, the average clustering coefficient, the diameter and the number of referrals. The network evolution includes five phenomena: add, in the network edge, to the point, edge and reconnection of the five operating. Network dynamics is reflected in the interaction between points and points, the change of the state of the point itself and the local interaction of each point, which leads to the emergence of some functions and behaviors of the whole network. Therefore, we can say that the connection between the two points is the starting point of the research of complex 
networks. Figure 1 is a variety of complex networks in the real world [7-9].

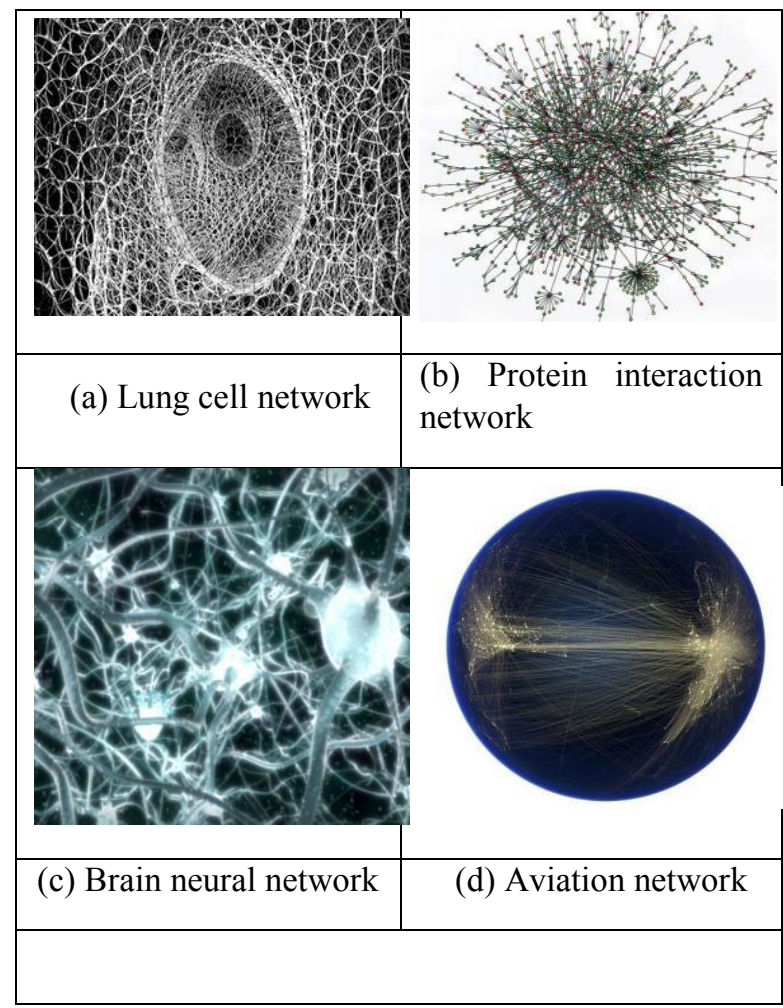

Fig.1. Four different types of complex networks

Although the scientific community for the complex network has not formed the unified definition of the concept, but the complexity of the network, as its name implies showing highly complex characteristics, mainly as follows.

(1) A complex composition: a large number of nodes and edges, which are composed of a variety of different features, and the network is often open, interacting with the outside world.

(2) Mixing Patters: nodes in complex networks can represent almost any individual with a certain characteristic, for example, the nodes in a complex network of actors represent different actors.

(3) The function diversity: the nodes in the network can choose the other nodes according to the principle of combining with certain attachment, the relationship among the nodes is very complex.

(4) Diversity of attributes: nodes and edges have different attributes, such as edges and weights of nodes are different, and it is possible to have the right of direction.

(5) Dynamic complexity: the edge set may belong to the nonlinear dynamics system, such as the edge of the state with the complex changes in time.
(6) Network evolution: the process of generating, growing, and dying of nodes or edges is very complicated. In the social relations network, the individual or group may or may not have the connection, which causes the relation network scale and the attribute to be difficult to determine.

(7) The fusion of multiple repeats: due to the complexity of the above interactions, it is difficult to grasp the changes of the complex network. For example, when the aircraft network planning and design, the size and volume of a single airport and the change of the weight of each route will change the topology of the entire network. More than one airport to open routes and adjust fares, passengers have more and more travel options, so that by constantly adjust the improvement and development of the evolution of the aviation network more and more optimized.

\section{B. Common Statistics of Complex Networks}

In complex networks, the distribution of the characteristic path length, degree and degree of the degree and the aggregation coefficient are the most basic properties of the complex network topology. The characteristic path length is one of the commonly used network invulnerability evaluation index, complex network invulnerability of common node degree and between as indicators of attack strategy. In order to facilitate the description, this paper takes the Underact Regular Simple Network as an example, the URSN network, as shown in Figure 2.

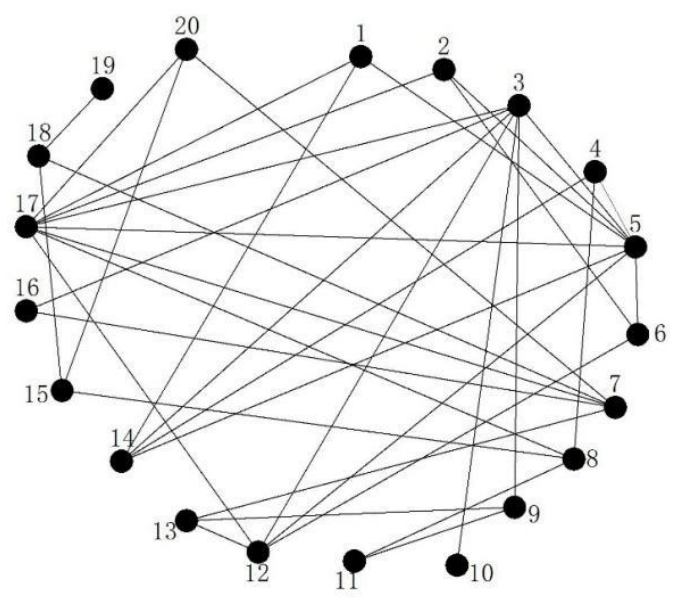

Fig.2. Node number is 20 URSN network

In the network, the number of edges that define the distance between two nodes is the shortest path to connect the two. The average value of the shortest path length between any two points is called the characteristic path length of the whole network (Characteristic Path Length), which is a kind of statistics which reflects the network size. If there is no path between the two points is considered that the path between the two points is infinite. The mathematical expression of the characteristic path length is 


$$
l=\frac{1}{N(N-1)} \sum_{i, j \in V, i \neq j} d_{i j}
$$

$l$ - Network characteristic path length;

$N$ - Network node number;

$V$ - Network node set

$d_{i j}$ - The shortest path length of node $\mathrm{i}$ to node $\mathrm{j}$.

The maximum characteristic path length between all nodes is known as the diameter of the network. The characteristic path length of URSN network is 2.3 , the diameter is 5 .

The number of connections between a node and the other nodes is the degree of the node (degree, with the letter K), which is a statistic describing the local features of the network. It is generally believed that the degree of the degree of the node is the important degree, that is, the greater the degree of nodes, the more important, the role of the network in the network, and vice versa. The degree distribution $P(k)$ means a random selection of a node in a network whose degree is equal to the probability of $k$. In order to calculate the URSN value and probability distribution network cases as shown in Table 2 and table 1 . The computation degree distribution is a very important statistic for analyzing the network characteristics. The distribution of the degree distribution is generally discrete, but in order to study the distribution regularity of its data point to be synthesized continuous function expression.

Table 1. URSN Degree value of network node

\begin{tabular}{|c|c|c|c|}
\hline Point & Degree & Point & Degree \\
\hline$k_{1}$ & 4 & $k_{6}$ & 3 \\
\hline$k_{2}$ & 3 & $k_{7}$ & 5 \\
\hline$k_{3}$ & 8 & $k_{8}$ & 4 \\
\hline$k_{4}$ & 3 & $k_{9}$ & 3 \\
\hline$k_{5}$ & 8 & $k_{10}$ & 1 \\
\hline$k_{11}$ & 2 & $k_{16}$ & 2 \\
\hline$k_{12}$ & 5 & $k_{17}$ & 8 \\
\hline$k_{13}$ & 3 & $k_{18}$ & 3 \\
\hline$k_{14}$ & 4 & $k_{19}$ & 1 \\
\hline
\end{tabular}

\begin{tabular}{|l|l|l|l|}
\hline$k_{15}$ & 3 & $k_{20}$ & 3 \\
\hline
\end{tabular}

Table.2. Probability distribution of URSN network nodes

\begin{tabular}{|c|r|r|r|r|r|r|}
\hline$k_{i}$ & 1 & 2 & 3 & 4 & 5 & 8 \\
\hline Frequency & 2 & 2 & 8 & 3 & 2 & 3 \\
\hline Frequency & $10 \%$ & $10 \%$ & $40 \%$ & $15 \%$ & $10 \%$ & $15 \%$ \\
\hline
\end{tabular}

In complex networks, if a node $i$ has a $m$ neighbor, then there is a maximum between the $m$ nodes, but it is actually only $h$. Then $m(m-1)$ is represented by $h$, which means that

$$
c_{i}=\frac{h}{n(n-1)}
$$

The aggregate coefficient of the network refers to the average value of all the nodes,

$$
\bar{c}=\frac{1}{N} \frac{h}{n(n-1)}
$$

The aggregate coefficients of each node are shown in Table 3.

Table.3. URSN Network node clustering coefficient

\begin{tabular}{|c|c|c|c|}
\hline Point & $\begin{array}{c}\text { Clustering } \\
\text { coefficient }\end{array}$ & Point & $\begin{array}{c}\text { Clustering } \\
\text { coefficient }\end{array}$ \\
\hline$c_{1}$ & 0.7655 & $c_{6}$ & 0.6667 \\
\hline$c_{2}$ & 0.6654 & $c_{7}$ & 0.7356 \\
\hline$c_{3}$ & 0.5449 & $c_{8}$ & 0.9712 \\
\hline$c_{4}$ & 0.3633 & $c_{9}$ & 0.8559 \\
\hline$c_{5}$ & 0.392857 & $c_{10}$ & 0 \\
\hline$c_{11}$ & 0 & $c_{16}$ & 0 \\
\hline$c_{12}$ & 0.4 & $c_{17}$ & 0.25 \\
\hline$c_{13}$ & 0 & $c_{18}$ & 0 \\
\hline$c_{14}$ & 0.666667 & $c_{19}$ & 0 \\
\hline$c_{15}$ & 0 & $c_{20}$ & 0.333333 \\
\hline
\end{tabular}


The average aggregate coefficient $\bar{c}=0.2446$.

The formula for defining a point is

$$
b(k)=\sum_{i \neq j, i, j \in N} \frac{m_{i j}(k)}{m_{i j}}
$$

Where $m_{i j}$ represents the shortest path between node $\mathrm{i}$ and node $\mathrm{j}, m_{i j}(k)$ represents the number of nodes $\mathrm{K}$ in all the shortest paths between points $\mathrm{i}$ and $\mathrm{j}, b(k)$ indicates the value of the node $k$, Later research shows that the number of the network has a very close relationship with the capacity of the network, if the greater the number of the network, the ability to synchronize the network is more weak. The number of nodes in URSN network is shown in Table 4.

Table.4. URSN Network node betweenness

\begin{tabular}{|c|c|c|c|}
\hline Point & Betweenness & Point & Betweenness \\
\hline$b_{1}$ & 0.072 & $b_{6}$ & 0.094 \\
\hline$b_{2}$ & 0.066 & $b_{7}$ & 0.027 \\
\hline$b_{3}$ & 0.039 & $b_{8}$ & 0.037 \\
\hline$b_{4}$ & 0.077 & $b_{9}$ & 0.063 \\
\hline$b_{5}$ & 0.089 & $b_{10}$ & 0.021 \\
\hline$b_{11}$ & 0.025 & $b_{16}$ & 0.058 \\
\hline$b_{12}$ & 0.043 & $b_{17}$ & 0.025 \\
\hline$b_{13}$ & 0.081 & $b_{18}$ & 0.087 \\
\hline$b_{14}$ & 0.018 & $b_{19}$ & 0.025 \\
\hline$b_{15}$ & 0.040 & $b_{20}$ & 0.047 \\
\hline
\end{tabular}

\section{NETWORK NODE IMPORTANCE EVALUATION}

\section{A. Definition of Node Importance}

There are obvious differences among the different nodes in the transportation network, which have obvious differences. Different nodes have different effects on the overall performance of the traffic network $[10,11]$. Because of the complexity of the complex network, the important degree of each node is different, and the complex network is represented by $G=(V, E)$. The adjacency matrix is $A=\left[a_{i j}\right]$, and several related concepts are defined before defining the network nodes.

(1) Node neighborhood

The neighborhood $\sigma_{k i}$ of node $v_{i}$ can be expressed as

$\sigma_{k i}=\left\{v_{j} \mid v_{j} \in V, a_{i j}=1, j=1,2, \ldots, n\right\}$

(2) Adjacency degree

The starting point of the network is $v_{i}$, and the shortest path length of $v_{j}$ is $d\left(v_{i}, v_{j}\right)$, where $N\left(v_{i}\right)$ is defined as the degree of the nodes

$$
N\left(v_{i}\right)=1 / \sum_{j=1}^{n} d\left(v_{i}, v_{j}\right) \quad i \neq j
$$

(3) Node criticality

Node $v_{i}$ the critical degree of $k\left(v_{i}\right)$ meaning: $v_{i}$ node degree $k$ neighborhood $\sigma_{k i}$, assuming that all nodes in after a number of shortest path node $v_{i}$ is $L\left(v_{i}\right)$, without after the number of shortest path node $v_{i}$ is $B\left(v_{i}\right)$, when $k \geq 2$, key to define the node $v_{i}$ degree for

$$
k\left(v_{i}\right)=\frac{L(i)}{B\left(v_{i}\right)+L(i)}
$$

When $k=1, k\left(v_{i}\right)=0$. In which $L\left(v_{i}\right)$ and $B\left(v_{i}\right)$ calculation method will be introduced. The greater the critical factor of the node, the more important the $\sigma_{k i}$ node is in the neighborhood of $k\left(v_{i}\right)$.

(4) Key domain of node

Key domain $F_{i}$ of the nodes

$$
F_{i}=\left\{v_{s} \mid v_{s} \in\left(\sigma_{g s} \bigcap_{\forall v_{s}, v_{t} \in \sigma_{k i}} \sigma_{g t}\right) \cup \sigma_{k i}\right\}
$$

The shortest path between two nodes $v_{s}$ and $v_{j}$ is $w_{i j}$, then $L\left(v_{i}\right)$ and $B\left(v_{i}\right)$ are calculated. 


$$
\begin{aligned}
& L\left(v_{i}\right)=\sum_{F_{i}} l\left(v_{i}\right) \quad l\left(v_{i}\right)= \begin{cases}1 / w_{i j} & v_{i} \in Q\left(v_{s}, v_{j}\right) \\
0 & v_{i} \notin Q\left(v_{s}, v_{j}\right)\end{cases} \\
& B\left(v_{i}\right)=\sum_{F_{i}} b\left(v_{i}\right) \quad b\left(v_{i}\right)= \begin{cases}0 & v_{i} \in Q\left(v_{s}, v_{j}\right) \\
1 & v_{i} \notin Q\left(v_{s}, v_{j}\right)\end{cases}
\end{aligned}
$$

The importance of nodes in the network can be expressed as

$$
R\left(v_{i}\right)=N\left(v_{i}\right) k\left(v_{i}\right)=\frac{L\left(v_{i}\right)}{\left[\sum_{j=1}^{n} d\left(v_{i}, v_{j}\right)\right]\left[B\left(v_{i}\right)+L\left(v_{i}\right)\right]}
$$

$N\left(v_{i}\right)$ - Node adjacency;

$k\left(v_{i}\right)$ - The key of node $v_{i}$;

$L\left(v_{i}\right)$ - The number of the shortest paths of the node $v_{i}$;

$B\left(v_{i}\right)$ - The shortest path number without node $v_{i}$.

The more important $R\left(v_{i}\right)$ is, the more important the nodes are in the network.

\section{B. Node Importance Evaluation Method}

The evaluation is helpful to effectively and accurately find the important nodes in the network,

Step 1. For $i=1$ to $n$;

Step 2. Computing the shortest path $d\left(v_{i}, v_{j}\right)$;

Step 3. According to the formula (3), the $N\left(v_{i}\right)$ of the node $v_{i}$ is calculated;

Step 4. Calculate the neighborhood $\sigma_{k i}$ and the key domain $F_{i}$ of node $v_{i}$;

Step 5. for (Neighborhood $\sigma_{k i}$ sigma all nodes $v_{i}, v_{j}$ ), all number is $1 / 2 k(k-1)$

(a) Find the shortest path set $Q\left(v_{s}, v_{j}\right)$

(b) According to (8) type to calculate $L\left(v_{i}\right)$

(c) According to (9) type to calculate $B\left(v_{i}\right)$

Step 6. According to (4) type to calculate $k\left(v_{i}\right)$;

Step 7. According to (10) type to calculate $R\left(v_{i}\right)$;
The algorithm the algorithm complexity depending in (2) and (5) two step, the known Dijkstra calculation node $v_{i}$ shortest path algorithm for complex degree for $O\left(n^{2}\right)$, algorithm in subsection (5) step of the algorithm complexity for $O\left(k^{3}\right)$, the algorithm complexity for $O\left(n^{3}+n k^{3}\right)$, while taking into account the in real network.

\section{IDENTIFICATION THEORY OF CRITICAL SECTION OF NETWORK CASCADING FAILURE}

\section{A. Critical Section Identification}

The ultimate goal of the research on the uncertainty analysis and the reliability of the road network is to obtain the road network. However, the existing researches on the reliability analysis and evaluation are more and more difficult to solve the problem of how to improve the reliability of road network [1214].

Road network reliability optimization design can usually be method comprises the following steps of: in after the formation of the road network traffic management stage, the research on road network reliability improvement play a larger role in the road network is a key component of the identification method, by means of key parts of the reinforcement to achieve the purpose of improving the reliability of the road network. Therefore, this chapter will focus on the two aspects of the research, one is based on the failure probability and the failure consequences of the key road identification method research, on the other hand, and it is considered that the importance of network nodes in the process of the cascading failure is studied.

Key sections are a key part of the road network, the identification of key sections of the road network for the rational allocation of network resources, meet the needs of different users, and thus improve the reliability of road network. With the continuous development of the uncertainty of the traffic system, the identification of key sections of the road network has caused the great attention of researchers.

The vulnerability of the road network can be described by the quadrant model shown in Figure 3, which has a high probability of failure and the large traffic network has a high vulnerability, while the low vulnerability of the traffic network has a low probability of failure and the failure effect is small. In this section, we use the concept of capacity random change road network as the research object, and propose a new method based on the failure probability and failure consequence. 


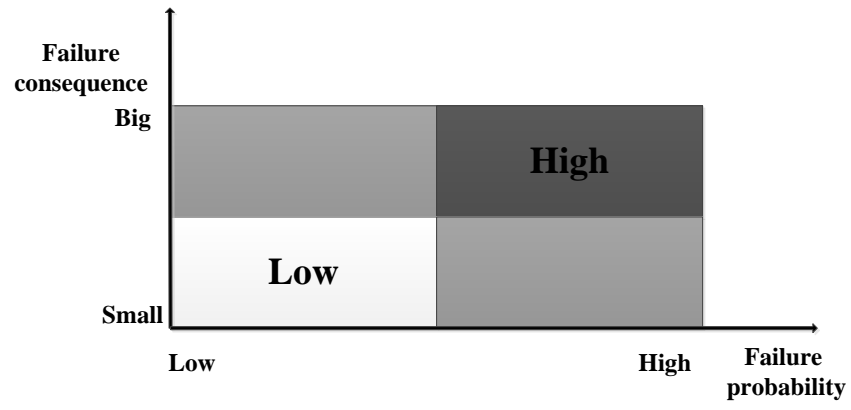

Fig.3. Road network vulnerability

\section{B. Critical Section Identification Method for Failure Probability and Consequence}

Assumed the capacity of the road section is uniform distribution,

$$
C_{a} \sim U\left(\theta_{a} C_{a}^{d}, C_{a}^{d}\right)
$$

The failure probability of a section $a$ is indicated by $p_{f, a}$.

The total running time in the maximum capacity is represented by $T T^{f, a},\left(T T^{f, a}-T T^{0}\right)$ said the failure of the section $a$.

Well, the identification model can be described as the critical section of the failure probability and the failure consequence [15-18].

$$
\begin{gathered}
I_{a}=p^{f, a} \times\left(T T^{f, a}-T T^{0}\right), \forall a \in A \\
T T^{0}=\sum_{a} t_{a}\left(x_{a}, c_{a}^{d}\right) \cdot x_{a}\left(c_{a}^{d}\right) \\
T T^{f, a}=\sum_{a} t_{a}\left(x_{a}, c_{a}^{d}\right) \cdot x_{a}, \frac{x a}{c a} \geq\left(\frac{V}{C}\right)_{t h}, \forall a \in A
\end{gathered}
$$

$I_{a}$ represent the key to the road $a ; x_{a}\left(c_{a}^{d}\right)$ represents the traffic flow in the maximum capacity state of the road $a$; $t_{a}\left(x_{a}, c_{a}^{d}\right)$ represents the travel time of the maximum capacity of the a section of the road $a ;\left(\frac{V}{C}\right)_{t h}$ indicates the failure criteria of the road $a$,If the saturation of the section a is satisfied by $\frac{x a}{c a} \geq\left(\frac{V}{C}\right)_{t h}$, It is considered that section of the road $a$ has failed.

A method of identifying the critical section of road network under stochastic supply is given, and the failure probability of a section is determined by Carlo Monte simulation. The traffic flow, road trip time and total impedance of a certain capacity state are determined by logit type random traffic assignment. The basic steps of the algorithm are as follows.

Step 1. Initialization. Set maximum sampling number $N_{\max }$. Make the sampling number $n=1$. Determine the failure criteria $\left(\frac{V}{C}\right)_{t h}$ of the road $a$. Initialization of the failure times $n_{a}=0$ of each section, The initialization section $a$ the consequence of failure $T T^{f, a}=0, \forall a$.

Step 2. The total operating time of the system is calculated. SUE traffic assignment is obtained for the maximum capacity status $c=\left(\cdots, c_{a}^{d}, \cdots\right)^{T}$.

Step 3. Sampling.

Using the random number generation method to generate the road capacity vector of the uniform distribution, the capacity of the road network $c^{(n)}=\left\{c_{1}^{(n)}, \cdots, c_{a}^{(n)}, \cdots, c_{|A|}^{(n)}\right\}^{T}$ under the condition of $n$ is obtained.

Step 4. Traffic assignment. For a specific section capacity vector $c^{(n)}$, SUE traffic assignment, Access to each section of traffic $x_{a}^{(n)}$, Road travel time $t_{a}^{(n)}$, Road saturation $\left(x_{a} / c_{a}\right)^{(n)}$, Road network travel time $T T^{(n)}$.

Step 5. Determine whether the road is invalid.

If $\left(x_{a} / c_{a}\right)^{(n)}>(V / C)_{t h}$, then make $n_{a}=n_{a}+1$, remember the consequences of failure a section $a$.

Step 6. To determine whether the maximum number of sampling times. If $n<N_{\max }$, then make $n=n+1$, Step into (3); Otherwise, step into (7).

Step 7. Calculate the key degree of each section of the road, press the key to calculate the key degree of each section.

$$
\begin{gathered}
p_{f, a}=\frac{n_{a}}{N_{\max }}, \forall a \in A \\
T T^{f, a}=\frac{T T^{f, a}}{n_{a}}, \forall a \in A \\
I_{a}=p_{f, a} \times T T^{f, a}, \forall a \in A
\end{gathered}
$$

\section{Analysis of Critical Section Identification Method for Failure Probability and Consequence}

As shown in Figure 4, the online number is a section number. The network consists of 13 nodes, 4 OD pairs, 19 sections and 25 paths. OD distribution is shown in Table 5, the characteristic 
parameters of road network, including the maximum capacity, free travel time and road section, are shown in Table 6 , and the connection paths between OD pairs are shown in Table 7.

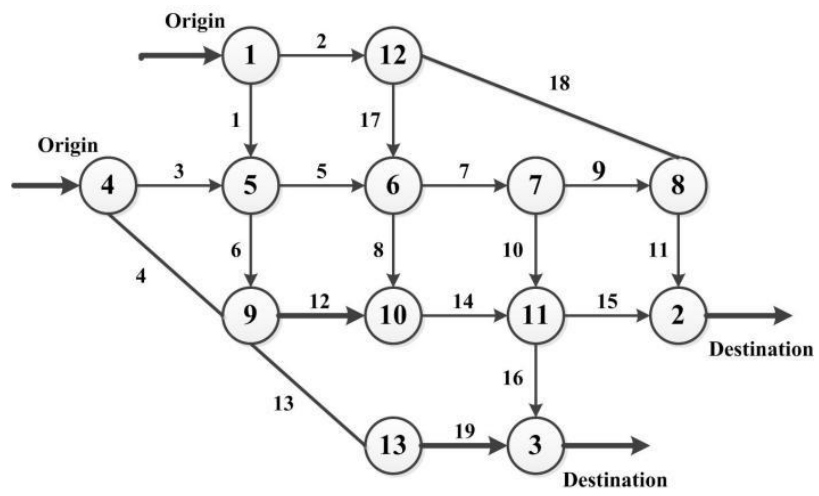

Fig.4. Example network

Table.5. OD distribution pattern $(\mathrm{pcu} / \mathrm{h})$

\begin{tabular}{|c|c|c|}
\hline$\theta$ & 2 & 3 \\
\hline 1 & 2500 & 2000 \\
\hline 4 & 2000 & 2500 \\
\hline
\end{tabular}

Table.6. Calculation example of network section characteristic parameters

\begin{tabular}{|c|c|c|c|c|c|}
\hline Section & $\begin{array}{l}\text { Free } \\
\text { flow } \\
\text { time }\end{array}$ & $\begin{array}{c}\text { Maximum } \\
\text { capacity } \\
(\mathrm{pcu} / \mathrm{h})\end{array}$ & Section & $\begin{array}{l}\text { Free } \\
\text { flow } \\
\text { time }\end{array}$ & $\begin{array}{c}\text { Maximum } \\
\text { capacity } \\
(\mathrm{pcu} / \mathrm{h})\end{array}$ \\
\hline 1 & 12 & 3000 & 11 & 12 & 3000 \\
\hline 2 & 12 & 3000 & 12 & 12 & 3000 \\
\hline 3 & 12 & 3000 & 13 & 24 & 3000 \\
\hline 4 & 24 & 3000 & 14 & 12 & 3000 \\
\hline 5 & 12 & 3000 & 15 & 12 & 3000 \\
\hline 6 & 12 & 3000 & 16 & 12 & 3000 \\
\hline 7 & 12 & 3000 & 17 & 12 & 3000 \\
\hline 8 & 12 & 3000 & 18 & 36 & 3000 \\
\hline 9 & 12 & 3000 & \multirow{2}{*}{19} & \multirow{2}{*}{12} & 3000 \\
\hline 10 & 12 & 3000 & & & 3000 \\
\hline
\end{tabular}
utilization factor on the importance of road section, $\theta_{a}=(0.95,0.90,0.85,0.80,0.75,0.65,0.60,0.55,0.50,0.40,0.30,0.20,0.70)$ were investigated. Figure 5 shows the capacity utilization factor of failure probability of each section. With the decrease of capacity utilization coefficient and the increase of failure probability of each road section, the fluctuation of traffic supply will increase, which will lead to the gradual failure of each road
To investigate the influence of different section capacity

\begin{tabular}{|c|c|c|}
\hline OD & Path number & Node sequence \\
\hline \multirow{8}{*}{$q_{12}$} & 1 & $1 \rightarrow 12 \rightarrow 8 \rightarrow 2$ \\
\hline & 2 & $1 \rightarrow 12 \rightarrow 6 \rightarrow 7 \rightarrow 8 \rightarrow 2$ \\
\hline & 3 & $1 \rightarrow 12 \rightarrow 6 \rightarrow 7 \rightarrow 11 \rightarrow 2$ \\
\hline & 4 & $1 \rightarrow 12 \rightarrow 6 \rightarrow 10 \rightarrow 11 \rightarrow 2$ \\
\hline & 5 & $1 \rightarrow 5 \rightarrow 6 \rightarrow 7 \rightarrow 8 \rightarrow 2$ \\
\hline & 6 & $1 \rightarrow 5 \rightarrow 6 \rightarrow 7 \rightarrow 11 \rightarrow 2$ \\
\hline & 7 & $1 \rightarrow 5 \rightarrow 6 \rightarrow 10 \rightarrow 11 \rightarrow 2$ \\
\hline & 8 & $1 \rightarrow 5 \rightarrow 9 \rightarrow 10 \rightarrow 11 \rightarrow 2$ \\
\hline \multirow{6}{*}{$q_{13}$} & 9 & $1 \rightarrow 12 \rightarrow 6 \rightarrow 7 \rightarrow 11 \rightarrow 3$ \\
\hline & 10 & $1 \rightarrow 12 \rightarrow 6 \rightarrow 10 \rightarrow 11 \rightarrow 3$ \\
\hline & 11 & $1 \rightarrow 5 \rightarrow 6 \rightarrow 7 \rightarrow 11 \rightarrow 3$ \\
\hline & 12 & $1 \rightarrow 5 \rightarrow 6 \rightarrow 10 \rightarrow 11 \rightarrow 3$ \\
\hline & 13 & $1 \rightarrow 5 \rightarrow 9 \rightarrow 10 \rightarrow 11 \rightarrow 3$ \\
\hline & 14 & $1 \rightarrow 5 \rightarrow 9 \rightarrow 13 \rightarrow 3$ \\
\hline \multirow{5}{*}{$q_{42}$} & 15 & $4 \rightarrow 5 \rightarrow 6 \rightarrow 7 \rightarrow 8 \rightarrow 2$ \\
\hline & 16 & $4 \rightarrow 5 \rightarrow 6 \rightarrow 7 \rightarrow 11 \rightarrow 2$ \\
\hline & 17 & $4 \rightarrow 5 \rightarrow 6 \rightarrow 10 \rightarrow 11 \rightarrow 2$ \\
\hline & 18 & $4 \rightarrow 5 \rightarrow 9 \rightarrow 10 \rightarrow 11 \rightarrow 2$ \\
\hline & 19 & $4 \rightarrow 5 \rightarrow 9 \rightarrow 11 \rightarrow 2$ \\
\hline \multirow{6}{*}{$q_{43}$} & 20 & $4 \rightarrow 5 \rightarrow 6 \rightarrow 7 \rightarrow 11 \rightarrow 3$ \\
\hline & 21 & $4 \rightarrow 5 \rightarrow 6 \rightarrow 10 \rightarrow 11 \rightarrow 3$ \\
\hline & 22 & $4 \rightarrow 5 \rightarrow 9 \rightarrow 10 \rightarrow 11 \rightarrow 3$ \\
\hline & 23 & $4 \rightarrow 9 \rightarrow 10 \rightarrow 13 \rightarrow 3$ \\
\hline & 24 & $4 \rightarrow 9 \rightarrow 10 \rightarrow 11 \rightarrow 3$ \\
\hline & 25 & $4 \rightarrow 9 \rightarrow 13 \rightarrow 3$ \\
\hline
\end{tabular}


section. The failure of each road section will gradually extend to $2,3,11,16$ road sections, and then to each road section of the road network. To simplify the analysis, the failure probability of large sections is taken to carry out important degree evaluation, when $\theta_{a}=0.2$ the failure probability is above 0.8 sections, including sections 14 , section 2 , section 11 , section 3 and section 5 .

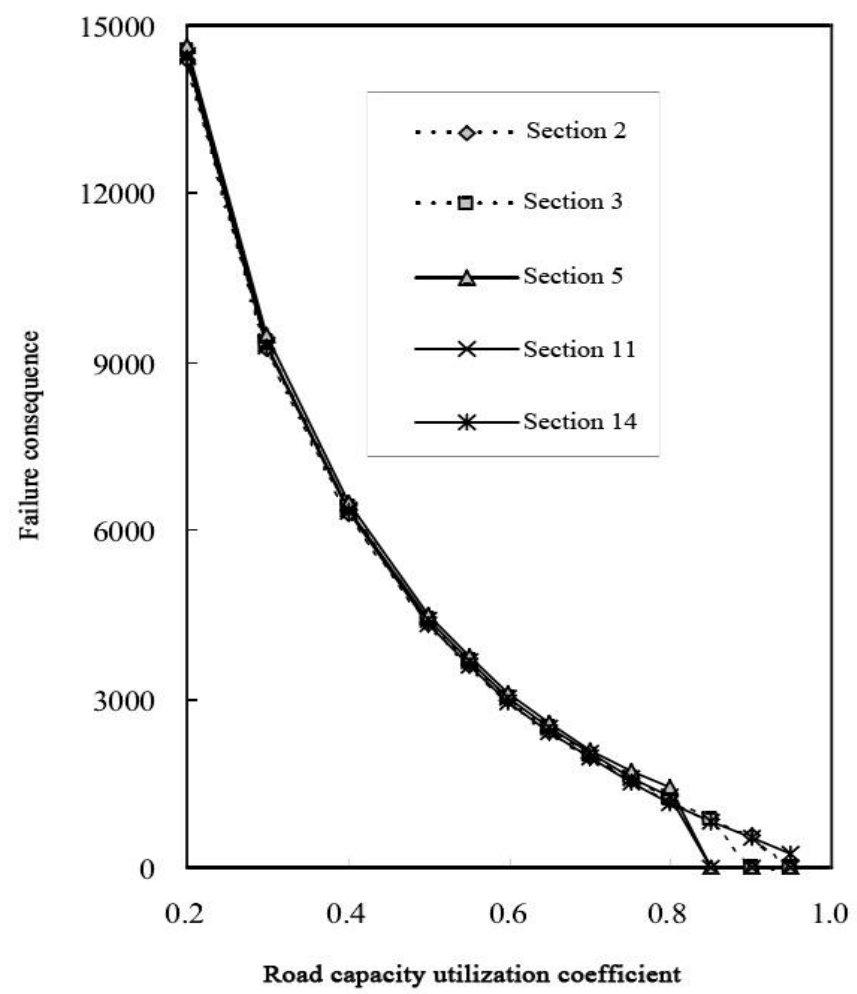

Fig.5. Failure consequences of different capacity utilization factors

It can be seen from Figure 5 that with the decrease of capacity utilization coefficient, the failure of road section is more and more serious, which indicates that the increase of road congestion will lead to the rapid increase of network impedance. The results of the initial failure state are similar to those of each section in the failure state, but the initial failure consequences of each section are greater than those of other sections. The abrupt change of road operation state has a great impact on the impedance of expressway network.

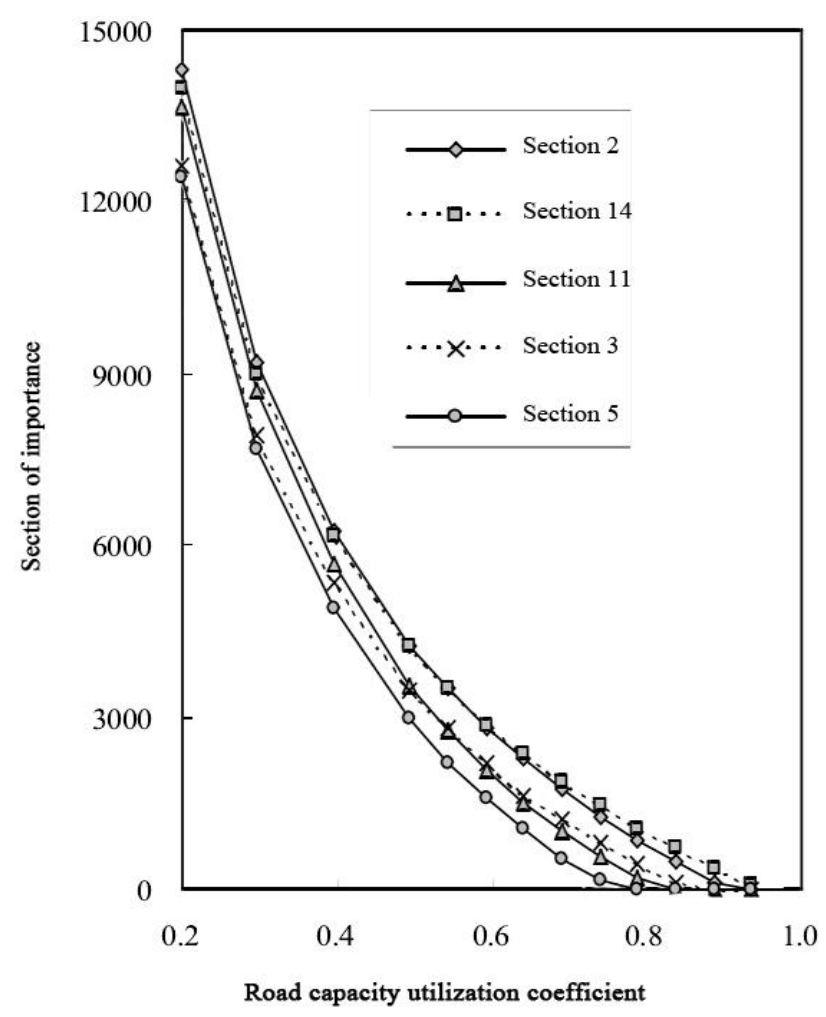

Fig.6. The importance of road section under different capacity utilization coefficient

It can be seen from Figure 6 that when the capacity utilization coefficient of road section is the largest, the importance of road section 14 is the most critical road section of the road network. With the further decline of the capacity utilization coefficient of road section, the importance of road section 2 becomes more prominent, and when the importance of road section 2 is the largest. Therefore, reasonable repair and reinforcement strategies should be formulated according to the degree of capacity degradation and the importance of different sections in practice.

Figure 5 and Figure 6 show the failure consequences of these sections and the coefficient of the link between the importance and the capacity utilization.

\section{IDENTIFICATION OF KEY NODES IN THE NETWORK CONSIDERING CASCADING FAILURE}

The interaction between the travel network and the road network, and the failure of the nodes is related. Therefore, the calculation of node importance should be considered simultaneously in these two networks. In this section, we will establish a double layer network model to characterize the hybrid network characteristics of the traffic network, and the importance of the nodes is studied. 
In real networks, a few nodes in the network have a failure, which can cause failure of other nodes through the connection of network nodes, which will result in a chain effect, which will eventually lead to the collapse of the whole network. This phenomenon is called failure cascading. The load of a node will be more than its capacity, the load of the fault node in accordance with a certain strategy to distribute to other nodes in the network, because of the additional load, the total load may exceed its capacity. This process is repeated, and the influence of the nodes gradually spread, resulting in cascading failure. Load - capacity cascading failure model is shown in Figure 7.

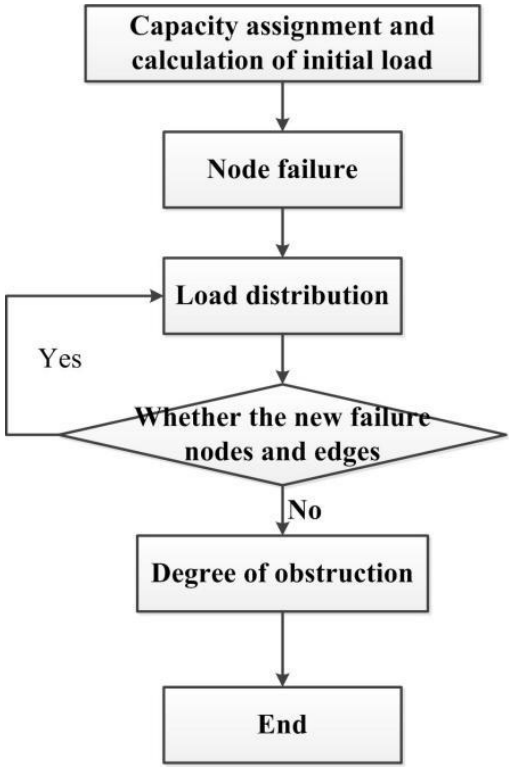

Fig.7. Load-Capacity cascading failure model

\section{A. Identification of Node Importance based on Cascading Failure}

The road network, which is shown in Figure 8, is composed of 25 nodes and 40 edges, and the network parameters are shown in Table 8. WS small world networks (network smallworld, SWN), BA free scale network structure algorithm are constructed respectively with 25 nodes of the travel network, respectively, as shown in Figure 8 and Figure 9. In WS algorithm, each node is connected to a $4 / 2$ node, which is connected with the left and right sides, and then to the edge of 0.5 . The average degree of nodes is 2 .

If the total demand is $100000 \mathrm{pcu} / \mathrm{h}$, the OD needs the same. Then, the small world travel network shown in Figure 9, OD for traffic are $1667 \mathrm{pcu} / \mathrm{h}$; the OD of the non scale travel network shown in Figure 10, for traffic are $3125 \mathrm{pcu} / \mathrm{h}$. According to the node importance in this section, we choose the travel network, which is shown in Figure 9 and Figure 10, respectively, to evaluate the importance of the road network in the cascading failure conditions, and to analyze the impact of the travel network structure on the node importance. The UE and SUE distribution models are used to compare the influence of different path selection behavior to the node importance in the same travel network.

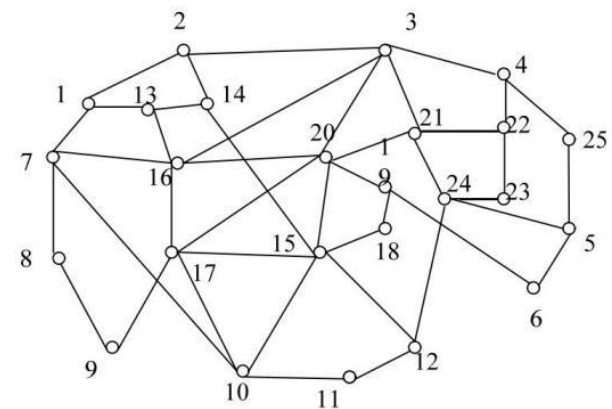

Fig.8. Road network topology diagram

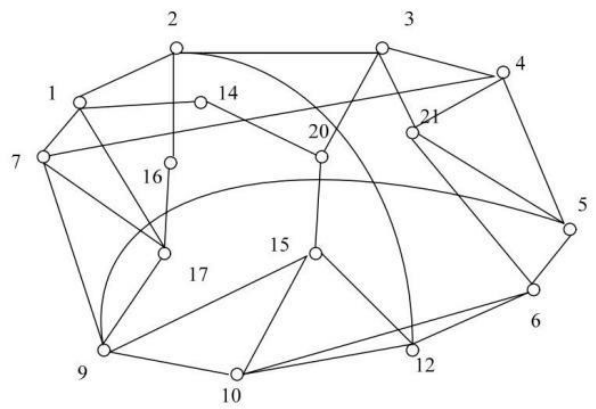

Fig.9. Small world network (SWN)

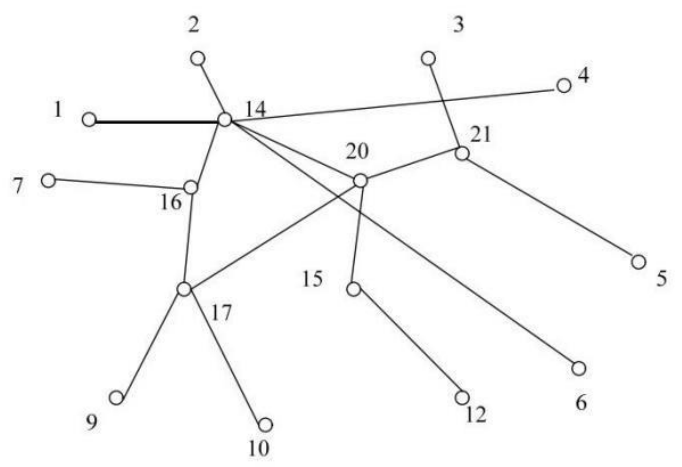

Fig.10. Scale free network (SFN)

Table 8. Free flow time and initial capacity of each section of road network

\begin{tabular}{|l|l|l|l|l|l|}
\hline Section & $\begin{array}{c}\text { Free } \\
\text { flow } \\
\text { time } \\
(\mathrm{s})\end{array}$ & $\begin{array}{c}\text { Initial } \\
\text { capacity } \\
(\mathrm{pcu} / \mathrm{h})\end{array}$ & $\begin{array}{c}\text { Sectio } \\
\mathrm{n}\end{array}$ & $\begin{array}{c}\text { Free } \\
\text { flow } \\
\text { time } \\
(\mathrm{s})\end{array}$ & $\begin{array}{c}\text { Initial } \\
\text { capacity } \\
(\mathrm{pcu} / \mathrm{h})\end{array}$ \\
\hline $1-2$ & 28 & 4000 & $15-18$ & 9 & 6000 \\
\hline $2-3$ & 33 & 4000 & $18-19$ & 7 & 6000 \\
\hline $3-4$ & 23 & 4000 & $15-20$ & 19 & 6000 \\
\hline
\end{tabular}


INTERNATIONAL JOURNAL OF CIRCUITS, SYSTEMS AND SIGNAL PROCESSING

\begin{tabular}{|l|l|l|l|l|l|}
\hline $4-25$ & 10 & 4000 & $3-16$ & 30 & 4000 \\
\hline $25-5$ & 7 & 4000 & $3-20$ & 21 & 6000 \\
\hline $5-6$ & 15 & 4000 & $3-21$ & 20 & 6000 \\
\hline $6-19$ & 13 & 6000 & $20-21$ & 14 & 6000 \\
\hline $19-20$ & 11 & 4000 & $21-22$ & 7 & 6000 \\
\hline $1-7$ & 15 & 4000 & $22-23$ & 4 & 2000 \\
\hline $7-8$ & 10 & 6000 & $23-24$ & 4 & 2000 \\
\hline $8-9$ & 24 & 6000 & $4-22$ & 10 & 6000 \\
\hline $7-10$ & 25 & 4000 & $5-24$ & 16 & 4000 \\
\hline $10-11$ & 17 & 4000 & $21-24$ & 7 & 6000 \\
\hline $11-12$ & 11 & 4000 & $12-24$ & 15 & 6000 \\
\hline $1-13$ & 17 & 2000 & $16-20$ & 33 & 6000 \\
\hline $13-14$ & 14 & 6000 & $17-20$ & 20 & 6000 \\
\hline $2-14$ & 13 & 6000 & $16-17$ & 10 & 4000 \\
\hline $14-15$ & 31 & 6000 & $13-16$ & 14 & 4000 \\
\hline $10-15$ & 22 & 6000 & $17-9$ & 12 & 6000 \\
\hline $12-15$ & 19 & 6000 & $10-17$ & 24 & 6000 \\
\hline $15-17$ & 26 & 6000 & $7-16$ & 21 & 6000 \\
\hline
\end{tabular}

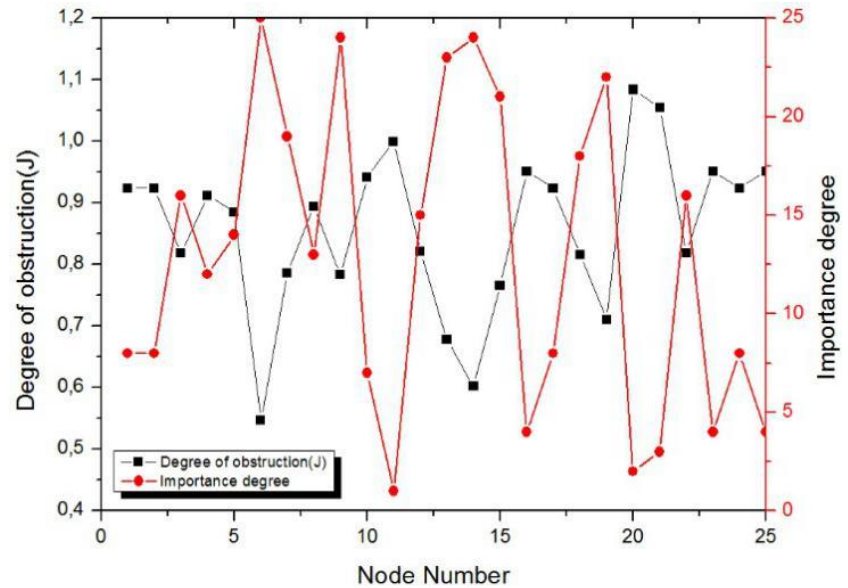

Fig.12. Results (SWN) under SUE distribution

When the user chooses the path according to the user, the results are shown in Figure 13 and Figure 14.

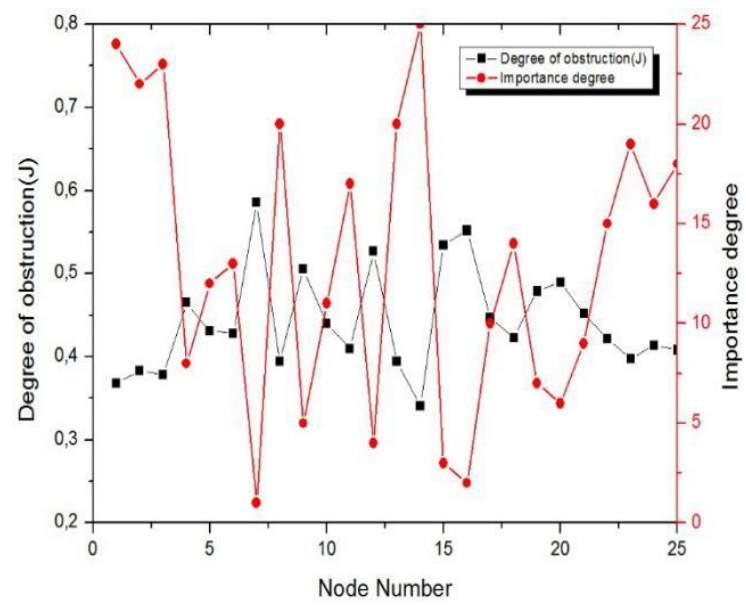

Fig.13. Results (SWN) under UE distribution

When the user chooses the path according to the user, the results are shown in Figure 11 and Figure 12.

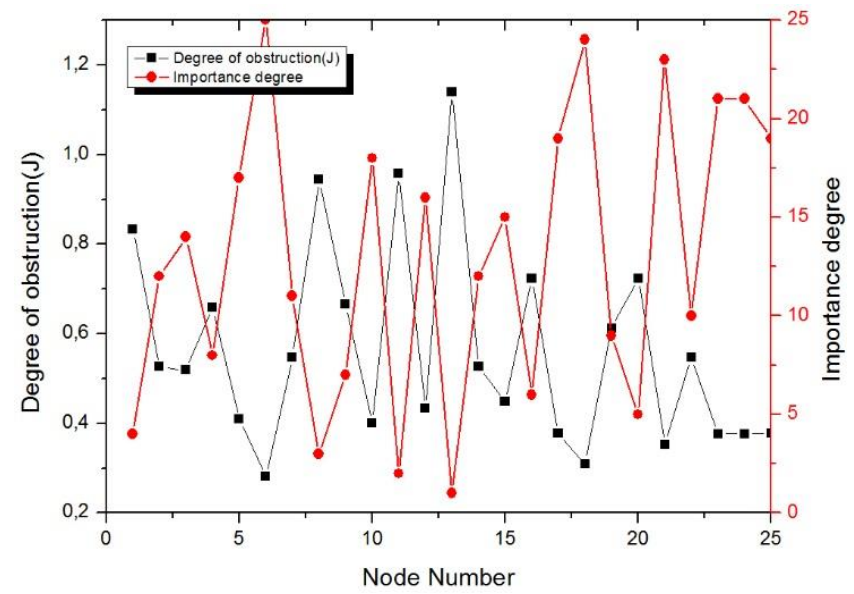

Fig.11. Results (SWN) under UE distribution

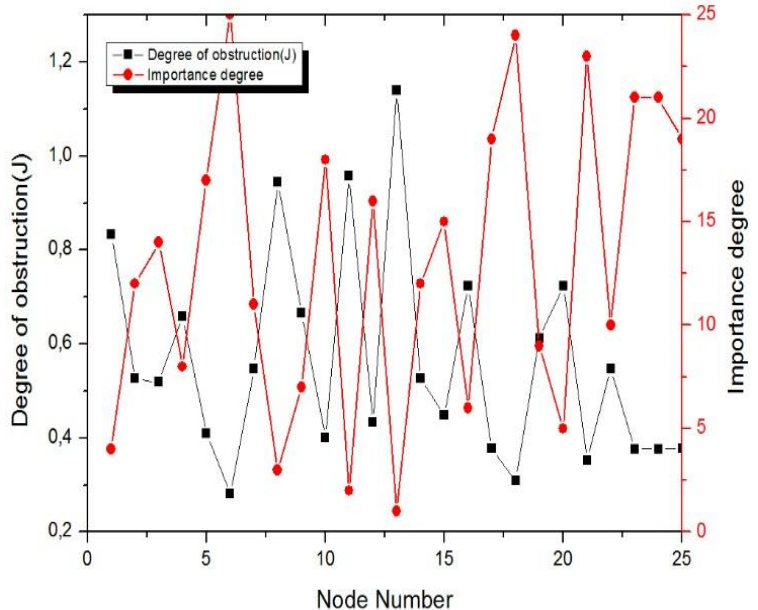

Fig.14. Results (SFN) under SUE distribution 


\section{Comparative Analysis}

The most important 4 nodes are selected from the graph 11 14. After deleting the 4 nodes, the blocking degree of the residual network is shown in Table 9.

Table 9. Analysis of the blocking degree of the 4 most important nodes

\begin{tabular}{|c|c|c|c|c|c|}
\hline \multicolumn{2}{|c|}{ Importance ranking } & 1 & 2 & 3 & 4 \\
\hline \multirow{4}{*}{ SWN } & UE distribution & 21 & 20 & 10 & 15 \\
\cline { 2 - 6 } & Degree of obstruction & 0.929 & 0.794 & 0.787 & 0.782 \\
\cline { 2 - 6 } & SUE distribution & 11 & 20 & 21 & 16 \\
\cline { 2 - 6 } & Degree of obstruction & $\infty$ & 1.084 & 1.055 & 0.951 \\
\hline \multirow{4}{*}{ SFN } & UE distribution & 7 & 16 & 15 & 12 \\
\cline { 2 - 6 } & Degree of obstruction & 0.586 & 0.552 & 0.534 & 0.527 \\
\cline { 2 - 6 } & SUE distribution & 13 & 11 & 8 & 1 \\
\cline { 2 - 6 } & Degree of obstruction & 1.139 & 0.958 & 0.944 & 0.833 \\
\hline
\end{tabular}

In the small world and the non-scale travel network, the important degree of each node is different. The travel route choice behavior has an important influence on the important degree of nodes, regardless of the structure of the travel network, UE and SUE path selection behavior has led to significant changes in the importance of nodes. For this example, the small world travel network, the attack of the key nodes caused by the degree of damage to large; if the structure of the travel is not subject to the network, it is conducive to system resistance to attack. If you choose to travel path in accordance with the SUE model, if the most important nodes to attack, a small world travel network will be paralyzed, and the road network under the no standard travel network is only part of the road is blocked, the system will not be paralyzed. In this example, the traveler in freeway network is more familiar, the road network against intentional attack ability is stronger, both in the small world of travel network is scale-free travel net, Walker if according to the UE to select the travel route, after the most important nodes suffer from intentional attack, the network blocking degree are small, in particular, no scaling travel network, even if the most important node is a deliberate attack, the road network is not blocked.

\section{CONCLUSION}

Whether the traffic system has the characteristics of complex network, the research results of the complex network has great application prospect. At the same time, it also can further expand and improve the complex network theory. In this paper, a new method of identifying the critical section of road network based on the failure probability and the failure consequence is established. The effect of capacity degradation degree on the failure probability, the failure consequence and the importance of the road section is analyzed. Complex network research object is a complex system, analysis and processing of complex network problems need to form a system of thinking. The supply and demand of the actual transportation network has a lot of uncertainty, and the uncertainty of the supply and demand of the traffic will be the risk of the individual trip decision and the road network construction management.

\section{ACKNOWLEDGEMENTS}

This work is supported by the MOE Layout Foundation of Humanities and Social Sciences, Research on engineering ethics education in Applied Universities under the background of new engineering construction, (No. 20YJA880064); Shanghai Educational Science Research Project, Research on the Integration Mechanism of Value Leading and Knowledge Teaching in Business Course Teaching under the Idea of Course Ideology and Politics, (No. C18048).

\section{REFERENCES}

[1] Yang X, GAO Z, Zhao X, et al. Road capacity at bus stops with mixed traffic flow in China [J]. Transportation Research Record: Journal of the Transportation Research Board, 2009 (2111): 18-23.

[2] Ge H X, Dai S Q, Dong L Y, et al. Stabilization effect of traffic flow in an extended car-following model based on an intelligent transportation system application [J]. Physical Review E, 2004, 70(6): 066134.

[3] Koshy R Z, Arasan V T. Influence of bus stops on flow characteristics of mixed traffic $[\mathrm{J}]$. Journal of transportation engineering, 2005, 131(8): 640-643.

[4] WANG Z, HUANG Z. An Analysis and Discussion on Short-Term Traffic Flow Forecasting [J]. Systems Engineering, 2003, 6: 019.

[5] Ran B, Boyce D. Dynamic urban transportation network models: theory and implications for intelligent vehicle-highway systems $[\mathrm{M}]$. Springer Science \& Business Media, 2012.

[6] Tolley R, Turton B J. Transport systems, policy and planning: a geographical approach [M]. Routledge, 2014.

[7] Chadwick G. A systems view of planning: towards a theory of the urban and regional planning process [M]. Elsevier, 2013.

[8] Hearnshaw E J S, Wilson M M J. A complex network approach to supply chain network theory $[\mathrm{J}]$. International Journal of Operations \& Production Management, 2013, 33(4): 442-469.

[9] Zhang L, Chang H, Xu R. Supernetwork Model of Enterprise Marketing Based on Complex Network Theory[C]//International Conference on Logistics Engineering, Management and Computer Science (LEMCS 2014). Atlantis Press, 2014.

[10] Sporns O. The human connectome: a complex network [J]. Annals of the New York Academy of Sciences, 2011, 1224(1): 109-125.

[11] Nazli Goker, Mehtap Dursun, and Elif Dogu, A Cognitive Mapping Approach for Evaluation of Success Indicators of IT Transformation Project, Engineering World, Volume 2, 2020, pp. 96-99.

[12] Chen Y, Hu A, Hu X. Evaluation method for node importance in communication networks [J]. J Chin INS, 2004, 8: 017.

[13] Dobson I, Carreras B A, Lynch V E, et al. Complex systems analysis of series of blackouts: Cascading failure, critical points, and selforganization [J]. Chaos: An Interdisciplinary Journal of Nonlinear Science, 2007, 17(2): 026103. 
[14] Harley Largo, Paulo J. G. Ribeiro, Gabriel Dias, Claudia P. B. Trujillo, Public Transportation Demand Model for Low Density Territories, WSEAS Transactions on Environment and Development, Volume 15, 2019, Art. \#43, pp. 395-407.

[15] Zongxiang L, Zhongwei M, Shuangxi Z. Cascading failure analysis of bulk power system using small-world network model[C]//Probabilistic Methods Applied to Power Systems, 2004 International Conference on. IEEE, 2004: 635-640.

[16] Tampère C, Stada J, Immers B, et al. Methodology for identifying vulnerable sections in a national road network [J]. Transportation Research Record: Journal of the Transportation Research Board, 2015.

[17] Mokhtarzade M, Zoej M J V, Ebadi H. Automatic road extraction from high resolution satellite images using neural networks, texture analysis, fuzzy clustering and genetic algorithms[C]//The international archives of the photogrammetry remote sensing and spatial information sciences 2008 Proceedings ISPRS Congress Beijing, B3b. 2008, 549.

[18] Taylor M A P, Sekhar S V C, D'Este G M. Application of accessibility based methods for vulnerability analysis of strategic road networks [J]. Networks and Spatial Economics, 2006, 6(3-4): 267-291.

\section{Creative Commons Attribution License 4.0 (Attribution 4.0 International, CC BY 4.0)}

This article is published under the terms of the Creative Commons Attribution License 4.0

https://creativecommons.org/licenses/by/4.0/deed.en_US 